\title{
A low-cost Home Automation System based on Power-Line Communication Links
}

\author{
E. Mainardi, S. Banzi, M. Bonfè, S. Beghelli
}

\begin{abstract}
In this paper we present a feasible Home Automation System scenario based on a very cheap distributed microcontroller architecture, rather than on devices interconnected by an expensive commercial bus. The means used for data communication is the home powerline, so that the system doesn't require placing other cables in addition to standard electrical facilities.
\end{abstract}

Index Terms- AC Powerline Commnication, Home Automation, Domotics, S.N.A.P. Protocol

\section{INTRODUCTION}

Building automation is now a day very widespread in all the industrialized countries, meaning with the term the application of automation and information technologies for the management of buildings like schools, hospitals, public edifices, private houses and so on. A younger sister of building automation is domotics, which is actually the application of the same techniques and tools in a domestic scenario, instead of a very big building. In this application domain, the cost, still very high, is the most important limit to the diffusion of these systems. Moreover, the lack in standardization and uniformity of communication protocols for home automation systems (i.e. Konnex-EIB®, MyHome BTicino®, Lonworks ${ }^{\circledR}$, to mention a few) is often a trouble for technicians required to design and install such plants, especially when the plant has to be installed in a pre-existing building whose electrical cabling is not prearranged to support that level of automation. In this case the solution proposed by the application of power line communication links (such as the U.S. X10 protocol) could be very interesting. These methods exploit standard electric lines (e.g. $220 \mathrm{~V} \mathrm{AC}, 50 \mathrm{~Hz}$ ) to transmit data, with specific modulation techniques, among the different modules (sensors, actuators, controllers) which are installed in the house. Standard protocols of this type are quite widespread in the United States, but their presence in European market is still quite limited. In this paper we present a practical implementation of a power line communication protocol, realized by means of Microchip PICmicro® microcontrollers.

E. Mainardi is with the Engineering Department, University of Ferrara, Ferrara, Italy (emainardi@ing.unife.it)

S. Banzi is with the Engineering Department, University of Ferrara, Ferrara, Italy (sbanzi@ing.unife.it)

M. Bonfè is with the Engineering Department, University of Ferrara, Ferrara, Italy (mbonfe@ing.unife.it)

S. Beghelli is with the Engineering Department, University of Ferrara, Ferrara, Italy (sbeghelli@ing.unife.it)
The first demonstrative application reported in the paper consists in the automatic opening and closing of windows in a domotic house and has been intentionally realized with minimal costs. Of course, this simple and low-cost system can be easily extended to manage every other service of the house (e.g. lights switching, air-conditioning, etc.). The higher-level communication protocol is based on the S.N.A.P. specification, which has been specifically developed for home automation, even if it is actually suitable for a wide variety of cases. The protocol is very scalable and is therefore very easy to implement either on low-cost microcontrollers, with the minimum of memory and computational resources, or on more complex and powerful devices.

\section{POWERLINE SYSTEMS}

Powerline systems use the standard electrical power lines to transmit data [1],[2].

They can be DC or AC powerline systems. The first ones transmit data over low DC voltages, typically $12-24 \mathrm{~V}$. They are exploited for smart-home scenarios in a few domotic-bus (such as KNX-EIB ${ }^{\circledR}$ and the Italian BTicino ${ }^{\circledR}$ ) and in smartsystems with a lot of sensors, (such as wearable network for health monitoring [3]) where is necessary to send data or to read the status without incrementing the number of cables [4].

AC powerline systems, instead, are applied over AC voltages both in closed environments (houses [5] but also hospitals, airports and so on [6]) and in the big distribution power networks, over very long distances and with big amount of power in play. In this last case AC powerline systems are able to offer several services such as reading the electricity meter, transmitting data and voice over the standard lines, paying the electricity bill, storing information about the network (peaks of power absorption, revealing tampering attempt), solving the "local-loop" problem (i.e. how to go from the optic fibre link that serves a city to every single home in the so called "last mile").

There are also some producers that allow choosing among different solution of the same system. For instance the KNXEIB association gives to the user the possibility to choose for its domotic-system between the standard bus (DC power line) and the home powerline (AC siystem) as a transmissive medium. 
Obviously the main advantage of the AC powerline systems is that there is no need to use other cables respect to those already in place for the power network.

Anyway, thinking about smart-home systems, if we consider AC or DC powerline, European or US standards (EIB ${ }^{\circledR}$ and BTicino ${ }^{\circledR}$ for the first one, the famous $\mathrm{X}-10{ }^{\circledR}[7],[8]$ for the second) the cost is the main problem for a private citizen who decided to install such a system in his home.

If we think about having just only a single communication link between two smart points, with a commercial solution one would spend at least a few hundreds euros (for KNX at least the DC power supply, the RS-232 interface to program the system and the binary output module are necessary, with Italian commercial X-10 products at least two X-10 tx-rx modules and a X-10 network controller, with BTicino at least the power supply module, a command module and an one relay actuator module)

\section{THE APPLICATION}

The aim of this work is to build a cheap powerline link between two stations using off the shelf components. The architecture is a simple master/slave: the master is the control unit, the one who is able to send commands such as "open window", "close window", "stop action". The slave is the unit that communicates with the window junction box, which acts the window motor to open, close and stop it. The user interface is a little keyboard with three buttons, each one for a different function, as shown in figure 1 .

\section{THE MODEM}

For our experiment we have used the PLM- $24^{\mathrm{TM}}$ power line modem [9] shown in figure 2. It is based on ST7537 Microtronics chip [10],[11] with FSK modulation and 2400 bps baud rate. The baud rate is not very high, but is perfectly compatible wih the human time involved in the application.

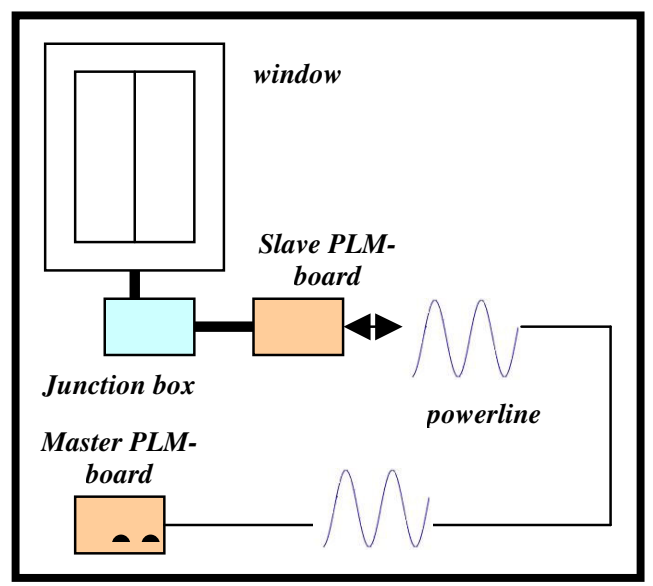

Fig.1. Overview of the whole system

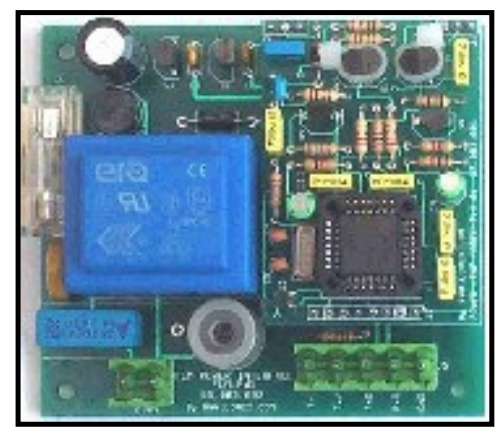

Fig.2. The PLM-24 power line modem

We have chosen a FSK module because, inside the CENELEC frequency band 9-148.5 KHz, it seems to be one of the more simple and robust modulation method for powerline communication [1], [12], [14]. Instead out of this band it seems that olny spread spectrum methods are possible [13].

In fact, since the power line carrier was not specifically designed for data transmission, it provides a harsh environment, and over such a transmission medium an amplitude modulation may be deeply disturbed (even if there are Ics that uses this modulation method, like the TDA5051)

In figure 3 is possible to view the TTL interface between the modem and the master units, where $\mathrm{rx}$ and tx are the two communications pin, $\mathrm{CD}$ is the carriage detection pin (it goes low when the line is busy) and RxITx is a pin which has to be put low when the modem is transmitting and high when the module is receiving.

The Microchip PIC16F876 is the microcontroller that manages the communication: if the user wants to send a command it transmits the associated packet over the network, detecting if someone else is carring the line or the line is free and acting a syncronization with the slave unit (for the syncronization method see [10]).

On the other side, the slave unit is sensing the power line to see if someone is transmitting. If so, after the syncronization phase, the microcontroller receives and processes the data to send the appropirate command to the window junction box (see Figure 5).

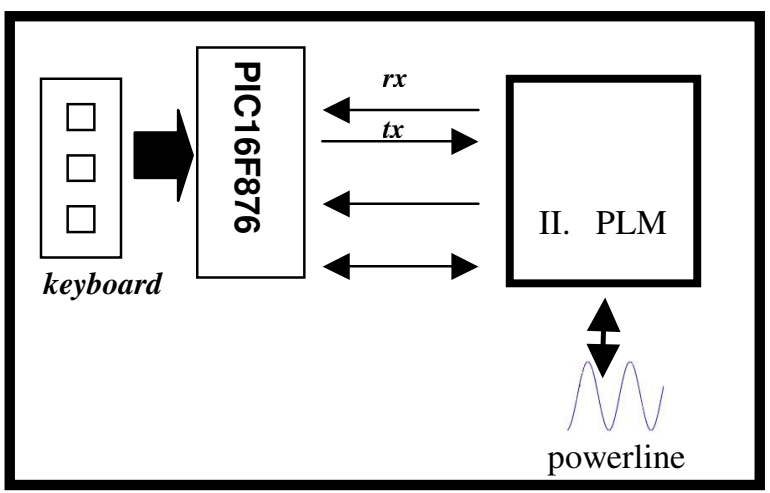

Fig. 3. Interface between powerline PLM24 modem and the master unit 


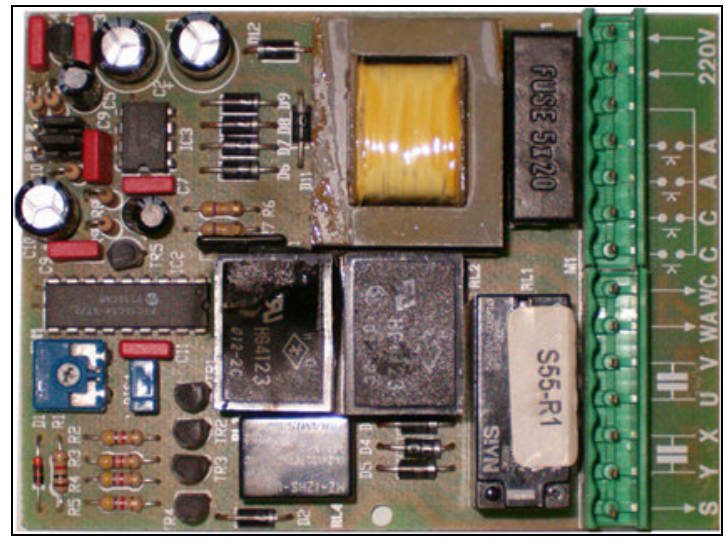

Fig. 4. The window junction box. The two $220 \mathrm{~V}$ pins are for the phase and neutral cable, the others are the output to the motor command and the input from a push-button control

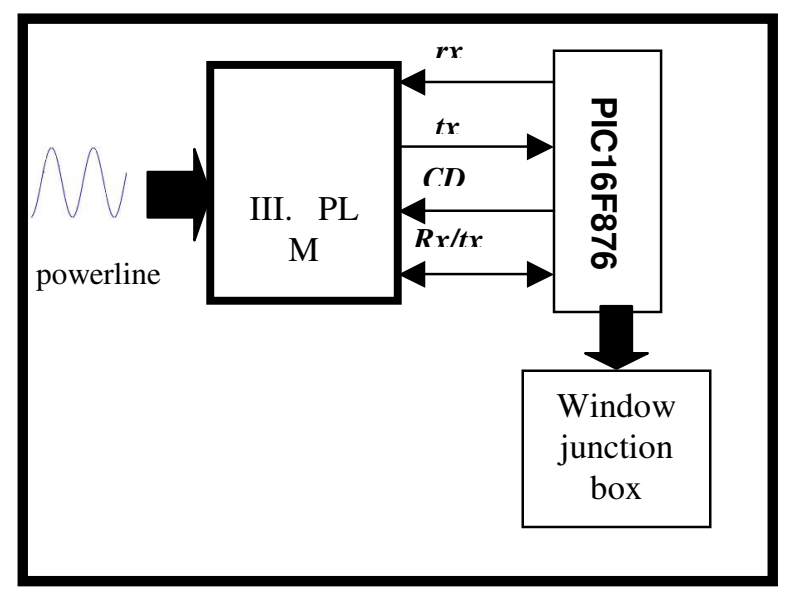

Fig. 5. The interface between the slave unit and the window junction box

\section{THE S.N.A.P. PROTOCOL}

The acronyms S.N.A.P. [15] stands for Scalable Node Address Protocol and represents a standard generic format for a packet transmission, no matter what is the phisical layer of the link. It has been defined specifically for home automation products, even if one can apply it to a lot of other applications. This free and open network protocol allows for different packet length and different protocol complexity, depending on the actual needs.

\begin{tabular}{|l|c|c|c|c|c|c|c|}
\hline SYNC & HD & HD & SA & DA & DATA & ED & ED \\
& 1 & 2 & & & & 1 & 2 \\
\hline
\end{tabular}

Fig. 6. The SNAP packet format

The packet has 1 byte of syncronization, 2 header bytes, up to 3 source and destination address bytes, up to 512 data bytes and up to 2 ED (Error Detection) bytes. The EDM (Error Detection Mode) can be specified by the user: FEC, 32-bit
CRC, 16-bit CRC, 8-bit CRC, 8-bit Checksum, 3 Times Retransmission and No Error Detection.

In our case we had one byte of source and destination address, 1 byte of data and one byte of ed. We implemented cheksum, CRC-8 (DOW CRC, $X^{8}+X^{5}+X^{4}+1$ ) and CRC-16 (CRC-CCITT $\left.X^{16}+X^{12}+X^{5}+1\right)$ edm [16],[17],[18]. The current used mode is cheksum.

The S.N.A.P. protocol allows also the use of ACK/NACK. Two bits are present in the header bytes to inform if the transmitter unit requires an acknoledge packet from the receiver.

In our case, since from the test the communication link has proved to be very robust, we haven't implemented this technique, but it is possible to do that in the future with minor software modifications.

\section{AVOIDING COLLISION}

To avoid collision a carriage detection method is implemented. The powerline modem has a CD pin that is tested when the user pushes a button to send a command to the slave unit. If the line is busy the command is stored in a circular buffer that keeps the packet until the line is free, otherwise the packet is istantanely sent. If the line is free the master takes it and holds it until the whole packet is transmitted.

There is a circular buffer also in the slave unit. It stores the received packets in case the user pushes a command button before the execution of the previous command has been completed. The two circular buffers have three elements each.

\section{CONCLUSION AND FUTURE WORK}

The aim of this work was to demonstrate that it is not necessary to spend a lot of money to build a home automation system, and that is possible to leave behind the troubles on choosing amomg a lot of possible commercial solution using very few, simple, cheap and off the shelf components. In fact with only 90 euros we have built a robust link between a master and a slave units, conversely of what would have been with a EIB, X-10 or BTicino solution.

Moreover, to extend the possibility of user interface, we are developing a remote control interface which communicates to the master unit via RF, and which is able to receive vocal commands. This future step will be also very cheep, being that the vocal unit (Sensory Voice Extreme) costs 40 euros (the commercial vocal systems such as "Easy by Voice" and "Sicare Pilot" cost more than 4000 euros!!!) and is easily connectable to a proprietary board with a microcontroller.

\section{REFERENCES}

[1] Anindya Majumder, James Caffery, Jr., "Power line communication: an overview", October/November 2004, IEEE Potentials 
[2] D. Clarke , "POWERLINE COMMUNICATIONS: Finally Ready for Prime Time?", January- February 1998, IEEE Internet Computing, Pages 10-11

[3] Eric R. Wade, H. Harry Asada, "DC Powerline Communication Network for a Wearable Health Monitoring System", Proceedings of ICRA '04. 2004 IEEE International Conference on Volume 4, April 26-May 1, 2004 Page(s):4085 - 4090 Vol.4

[4] Chun-Hung Liu, Eric Wade and H. Harry Asada , "Reduced-Cable Smart Motors Using DC Power Line Communication", Proceedings of the 2001 IEEE International Conference on Robotics \& Automation Seoul, Korea. May 21-26, 2001

[5] Yu-Ju Lin, Haniph A. Latchman, Minkyu Lee , "A Power Line Communication Network Infrastructure For The Smart Home", IEEE Wireless Communications • December 2002

[6] Er Liu, Yangpo Gao, Golam Samdani, Omar Mukhtar and Timo Korhonen, "Powerline Communication over Special Systems", Power Line Communications and Its Applications, 2005 International Symposium on 6-8 April, 2005 Page(s):167 - 171

[7] Dave Rye, "The X-10 Powerhouse, Power Line Interface", [Online], Aavailable: http://www.hometoys.com/

[8] Phil Kingery, "Home Toys Article- Digital X-10", - Feb99 - [Online], Available: http://www.hometoys.com

[9] PLM-01- PLM-24 Power Line Modem, User's manual and assembly guide, [Online], Available: http://www.lorix.it/

[10] J. Huloux, L. Hanus, "ST7537 Power Line Modem Application", SGSThomson, Application Notes [Online], Available: http://www.lorix.it/

[11] ST7535HS1 Home Automation Modem, Datasheet, June 1995, SGSThomson, [Online], Available: www.st.com

[12] Matthias Karl, Klaus Dostert, "Selection Of An Optimal Modulation Scheme For Digital Communications Over Low Voltage Power Lines", , Spread Spectrum Techniques and Applications Proceedings, 1996., I

[13] Petra Cuntic, Alen Baiant, "Analysis of Modulation Methods for Data Communications over the Low-voltage Grid", Proceeding of the $7^{\text {th }}$ International Conference on Telecommunication - ConTEL 2003 - June 11-13, Zagreb, Croatia

[14] Hakki Cavdar ,"Performance Analysis of FSK Power Line Communications Systems Over the Time-Varying Channels:Measurements and Modeling", IEEE Transactions On Power Delivery, Vol. 19, No. 1, January 2004

[15] S.N.A.P. Scaleable Node Address Protocol, (C) 1998-2002 HTH, Document revision 1.03, Document revision 1.03, (C) 1998-2002 HTH, Document revision 1.03, [Online], Available: http://www.hth.com/snap/

[16] Ross N. Williams, "A painless guide to crc error detection algorithms" 1996, , Rocksoft(tm) Pty Ltd, [Online], Available: http://www.on.net/clients/rocksoft/rocksoft/

[17] Tenkasi V. Ramabadran, Sunil S. Gaitonde, "A Tutorial on CRC Computations" , Micro, IEEE, Volume 8, Issue 4, Aug. 1988 Page(s): $62-75$

[18] P. Koopman,T. Chakravarty , "Cyclic Redundancy Code (CRC) Polynomial Selection For Embedded Networks", Proceedings of the 2004 International Conference on Dependable Systems and Networks (DSN'04) 